\title{
Role of chromatin remodeling complex SWI/SNF and VDR in chronic rhinosinusitis
}

\author{
Katarzyna Kowaliki,B-F, Martyna Waniewska-Łęczycka ${ }^{1, B, C, E, F}$, Elżbieta Sarnowska ${ }^{2, A-C, E, F}$, \\ Natalia Rusetska ${ }^{2, B, C, E, F}$, Janusz Sierdziński ${ }^{3, C, F}$, Mariola Zagor, ${ }^{1, A-C, E, F}$ \\ ${ }^{1}$ Department of Otorhinolaryngology, Faculty of Medicine and Dentistry, Medical University of Warsaw, Poland \\ 2 Department of Molecular and Translational Oncology, Maria Skłodowska-Curie Institute Oncology Center, Warszawa, Poland \\ ${ }^{3}$ Department of Medical Informatics and Telemedicine, Medical University of Warsaw, Poland \\ A - research concept and design; $\mathrm{B}$ - collection and/or assembly of data; $\mathrm{C}$ - data analysis and interpretation; \\ $D$ - writing the article; $E$ - critical revision of the article; $F$ - final approval of the article
}

\author{
Address for correspondence \\ Mariola Zagor \\ E-mail: popkom@interia.pl \\ Funding sources \\ None declared \\ Conflict of interest \\ None declared

\section{Acknowledgements} \\ We thank M. Tomaszewska for technical support
}

and J. Siedlecki for intellectual input.

Received on November 12, 2019

Reviewed on November 20, 2019

Accepted on January 30,2020

Published online on March 24, 2020

Cite as

Kowalik K, Waniewska-Łęczycka M, Sarnowska E, Rusetska N,

Sierdziński J, Zagor M. Role of chromatin remodeling complex SWI/SNF and VDR in chronic rhinosinusitis. Adv Clin Exp Med. 2020;29(3):313-323. doi:10.17219/acem/117683

DOI

10.17219/acem/117683

\section{Copyright}

Copyright by Author(s)

This is an article distributed under the terms of the

Creative Commons Attribution 3.0 Unported (CC BY 3.0)

(https://creativecommons.org/licenses/by/3.0/)

\begin{abstract}
Background. The SWI/SNF (SWItch/sucrose non-fermentable) chromatin remodeling complex enables glucocorticoid receptor (GR) and vitamin D receptor (VDR) to function correctly and is engaged in inflammation response. The SWI/SNF may play an important role in chronic rhinosinusitis (CRS).
\end{abstract}

Objectives. The aim of this study was to assess the following: 1) the gene and protein expression of the SWI/SNF complex subunits in sinonasal mucosa; 2) relation of SWI/SNF complex and VDR expression; and 3) correlation with clinical data.

Material and methods. The study population consisted of 52 subjects with CRS without nasal polyps, 55 with CRS with nasal polyps and 59 controls. The SWI/SNF protein expression level was analyzed in immunohistochemical (IHC) staining. Human nasal epithelial cells (HNECS) was stimulated using lipopolysaccharide (LPS), Staphylococcal enterotoxin B (SEB) and vitamin D3 (vitD3) in vitro. The transcript level of the SWI/SNF subunits was measured with polymerase chain reaction (PCR).

Results. In the control group, the intensity of the IHC staining for SWI/SNF subunits was significantly higher than in both groups of patients with CRS $(p<0.05)$. A positive correlation of the SWI/SNF protein expression was noticed with VDR expression level $(p<0.043)$. Association between SWI/SNF protein expression level and allergy, neutrophils and body mass index (BMI) has been observed $(p<0.05)$. The decreased transcript level of the SWI/SNF subunits genes in HNECs was observed after LPS stimulation and increased after vitD3 stimulation.

Conclusions. The SWI/SNF complex may influence CRS through steroid hormone signaling and VDR. Thus, modification in therapy may be mandatory in patients with CRS and altered SWI/SNF signaling, reflecting resistance to steroids treatment.

Key words: chronic sinusitis, nasal polyps, vitamin D, steroids 


\section{Background}

Chronic rhinosinusitis (CRS) is a heterogeneous disease characterized by symptomatic inflammation of paranasal sinuses and nasal cavity. Its prevalence is estimated at $14 \%$ in the American and $10.9 \%$ in the European population, and it is one of the most common human chronic diseases. ${ }^{1,2}$ The pathophysiology of CRS is multifactorial. Various theories on the etiology, such as allergy, bacterial and fungal infections, as well as structural abnormalities, have been proposed; however, the pathogenesis remains largely unknown. ${ }^{3}$

The CRS is typically classified in 2 phenotypes including CRS with nasal polyps (CRSwNP) and CRS without nasal polyps (CRSsNP). Symptoms in CRSwNP are associated more closely with clinical complaints of nasal obstruction and olfactory loss. The CRSwNP is more often combined with comorbidities such as asthma and aspirin hypersensitivity (this phenomenon is named Samter's triad). Moreover, CRSwNP tissues are characterized by more intense eosinophilic infiltration and a Th2-based cytokine profile. The CRSsNP tissues have been infiltrated mostly by neutrophils and Th1 cytokines. However, the distinct role of Th1/Th2 profiles in the subtypes of CRS gives rise to some controversy. ${ }^{4}$

Current medical knowledge does not explain in a detailed way the pathomechanism of CRS. However, over the past 20 years, the development of science in the field of immunology and molecular biology has allowed, at least in part, to understand the various pathophysiological processes taking place at the cellular level and involved in CRS. ${ }^{1,5}$

Regardless of the pathomechanism leading to CRS formation, the characteristic features of this disease are inflammation and tissue remodeling. One of the regulators of the inflammatory response is the SWI/SNF (SWItch/ sucrose non-fermentable) complex. Studies show that after lipopolysaccharide (LPS)-induced inflammation in macrophages, the SWI/SNF complex is necessary for the antiinflammatory response. ${ }^{6}$ The SWI/SNF is a multi-protein complex (15-20 subunits) that has the ability to provide a DNA sequence to the transcriptional apparatus. The SWI/SNF consists of the following: the core part, formed by 1 of the 2 ATP-ases BRG1 and BRM, which hydrolyze ATP, bind acetylated histones, regulate transcription and act as tumor suppressors; BAF155, BAF170, and INI-1 subunits that stabilize the core of the complex ${ }^{7,8}$; and a range of external proteins responsible for joining the complex in a tissue-specific manner.

For the first time, the SWI/SNF type chromatin remodeling complex was identified in the yeast Saccharomyces cerevisiae. ${ }^{9}$ Among the many processes regulated by the SWI/SNF complex, it has been shown that it is involved in the regulation of gene expression encoding the regulators of many different processes in the cell, such as adhesion, differentiation, hormonal response, and cell cycle. ${ }^{8,10}$ Further studies on the function of this complex will help us to better understand the mechanisms of tissue remodeling resulting from chronic inflammation.
The treatment of CRS should be started with pharmacological treatment and then - if there is no improvement in the clinical condition of the patient - surgery should be considered. The pharmacotherapy of CRS is primarily aimed at limiting inflammation. The drugs of first choice, in this case, are glucocorticosteroids (GS). ${ }^{2}$ They work by reducing neutrophil accumulation in the inflammatory foci; in addition, they reduce the production of inflammatory mediators by inhibiting the release of arachidonic acid from cell membranes, decreasing the permeability of blood vessels and limiting the production of secretions through the mucous glands. ${ }^{11}$

The SWI/SNF complex may play a significant role in the development and treatment of CRS. The subunits of the chromatin SWI/SNF remodeling complex interact directly with the glucocorticoid receptor (GR) and additionally regulate the expression of the GS response genes through binding to repeating DNA sequences called hormone response elements (HREs). ${ }^{12,13}$ Therefore, proper function of GR depends on the SWI/SNF complex, which determines the regulation of GS-dependent genes expression. Additionally, the latest findings indicate that the SWI/SNF complex is able to promote anti-inflammation processes in combination with activation of vitamin $D$ receptor (VDR). ${ }^{14}$ The role of vitamin $\mathrm{D}$ and its receptors has been vastly addressed in recent studies on CRS pathophysiology. Authors present their crucial role in regulation of the immune function in paranasal sinuses. ${ }^{15-19}$

\section{Objectives}

In the present study, we investigated: 1) the protein expression of the main SWI/SNF subunits (BAF155, BRM and BRG1) in sinonasal mucosa of patients with CRSwNP and CRSsNP, and in control group (CG); 2) gene expression of the SWI/SNF subunits in human nasal epithelial cells (HNECs) after treating with LPS, SEB and vitamin D3 (vitD3) in vitro; 3 ) correlation of obtained results with clinical data, e.g., allergy and steroids therapy data, and with VDR expression in sinonasal mucosa.

\section{Material and methods}

The study was conducted in accordance with the ethical standards of the Local Ethics Committees of Warsaw Medical University, Poland, approval No. KB/209/2016, and with the Helsinki Declaration. All participants signed informed consent.

\section{Material}

The study population consisted of 166 patients (63 females and 103 males) operated in the Ear, Nose and Throat (ENT) Department at the Faculty of Medicine and 
Dentistry of the Medical University of Warsaw. Patients were divided into 3 groups (Table 1). Study groups with CRSsNP and CRSwNP were distinguished on the basis of the interview, physical examination including endoscopic examination of nasal cavities, and paranasal sinuses computed tomography (CT) scan according to the clinical criteria of EPOS 2012. ${ }^{2}$ A CG included patients with anatomical abnormalities of nasal structures without additional inflammation in nasal cavity and paranasal sinuses.

All patients with CRS underwent a 3-month conservative therapy (adequate medical therapy (AMT)). ${ }^{2}$ Then, after ineffective AMT, the patients were qualified for surgical treatment.

The study material in all groups was collected from the ostiomeatal complex region during endoscopic nasal/sinus surgery, fixed in formalin and embedded in paraffin blocks:

- group 0 - CG (59 patients) fragments of healthy nasal mucous membrane (the ostiomeatal complex region) taken from patients undergoing endoscopic nasal surgery due to the non-inflammatory nasal diseases (septal deviation, anatomy variations of the lateral nasal wall - concha bullosa);

- group 1 - CRSsNP group (52 patients) fragments of the mucous membrane (the ostiomeatal complex region) taken from patients undergoing an endoscopic sinus surgery due to CRSsNP;

- group 2 - CRSwNP group (55 patients) fragments of the mucous membrane (the ostiomeatal complex region) taken from patients undergoing an endoscopic sinus surgery due to CRSwNP.

Exclusion criteria were the following: sinonasal tumor, external sinus surgery in the past, systemic disease (cystic fibrosis, sarcoidosis, autoimmune disease), fungal rhinosinusitis, and possibility of pregnancy.

\section{Clinical data}

All patients underwent CT of the paranasal sinuses, assessed according to the CT Lund-Mackay scale (L-M), and completed a questionnaire for the evaluation of sinus complaints SNOT-22 (Sino-Nasal Outcome Test). ${ }^{2}$ Data on allergy status were collected based on a medical interview, a skin prick test and total immunoglobulin $\mathrm{E}$ (IgE) levels in the blood. Bronchial asthma was determined according to Global Initiative for Asthma (GINA) 2015 criteria. ${ }^{20}$ Moreover, during ENT examination, CRS patients were assessed according to the Lund-Kennedy scale $(\mathrm{L}-\mathrm{K}) .{ }^{21}$ Complete blood count was analyzed in order to calculate the blood cells, especial eosinophils, and neutrophils in all examined groups (ALAB laboratory, Warszawa, Poland).

\section{Immunohistochemistry}

Immunohistochemical staining was performed on 3.5-micrometer sections of tissue sheared from paraffin blocks. The assay was performed in all examined patients. The study was carried out using the EnVision FLEX + detection system, Mouse, High pH Detection System (Dako, Glostrup, Denmark). After deparaffinization in xylene, the slides were rehydrated and incubation was carried out with optimal dilutions of antibodies: anti-SMARCC1/BAF155 (1:200, per $1 \mathrm{~h}$ in $25^{\circ} \mathrm{C}$ ) monoclonal antibodies (D7F8S) (Cell Signaling Technology, Danvers, USA), BRG1 (1:100, per $12 \mathrm{~h}$ in $5^{\circ} \mathrm{C}$ ) (G-7) (Santa Cruz Biotechnology, Santa Cruz, USA) and BRM (1:200, per $1 \mathrm{~h}$ in $25^{\circ} \mathrm{C}$ ) (D9E8B) XP (Santa Cruz Biotechnology) - forming part of the chromatin remodeling complex SWI/SNF type. The colored

Table 1. Patients' characteristics

\begin{tabular}{|c|c|c|c|c|c|}
\hline Characteristic variable & CG $(n=59)$ & CRSsNP $(n=52)$ & CRSwNP $(n=55)$ & Statistical analysis & $\mathrm{p}$-value \\
\hline $\begin{array}{l}\text { Age } \\
\text { range } \\
\text { average }\end{array}$ & $\begin{array}{c}19-70 \\
36.3\end{array}$ & $\begin{array}{c}18-77 \\
42.5\end{array}$ & $\begin{array}{c}22-83 \\
50.1\end{array}$ & $K-W, X^{2}=20.66$ & $<0.001$ \\
\hline $\begin{array}{l}\text { Sex } \\
\text { female } \\
\text { male }\end{array}$ & $\begin{array}{l}24 \\
35\end{array}$ & $\begin{array}{l}20 \\
32\end{array}$ & $\begin{array}{l}19 \\
36\end{array}$ & $x^{2}=0.46$ & 0.79 \\
\hline Average Lund-Kennedy scores & - & 4.8 & 8.9 & $K-W, X^{2}=55.5$ & $<0.001$ \\
\hline Average CT Lund-Mackay scores & 0.7 & 6.9 & 16.5 & $K-W, X^{2}=140.28$ & $<0.001$ \\
\hline Average SNOT-22 & 1.23 & 1.61 & 1.59 & $K-W, X^{2}=14.21$ & $<0.001$ \\
\hline Asthma & 1 & 1 & 18 & $x^{2}=13.58$ & $<0.008$ \\
\hline Allergy & 12 & 18 & 23 & $x^{2}=14.76$ & $<0.006$ \\
\hline $\begin{array}{l}\text { Eosinophil count } \\
\text { range } \\
\text { average }\end{array}$ & $\begin{array}{c}0.02-0.47 \\
0.14\end{array}$ & $\begin{array}{c}0.02-0.80 \\
0.21\end{array}$ & $\begin{array}{c}0.01-1.59 \\
0.41\end{array}$ & $K-W, X^{2}=34.36$ & $<0.001$ \\
\hline $\begin{array}{l}\text { Neutrophil count } \\
\text { range } \\
\text { average }\end{array}$ & $\begin{array}{c}2.04-9.80 \\
4.56\end{array}$ & $\begin{array}{c}1.01-9.24 \\
4.03\end{array}$ & $\begin{array}{c}1.08-11.35 \\
4.73\end{array}$ & $K-W, X^{2}=6.59$ & $<0.037$ \\
\hline
\end{tabular}

CG - control group; CRSsNP - patients with chronic rhinosinusitis without nasal polyps; CRSwNP - patients with chronic rhinosinusitis with nasal polyps. The data was assessed according to the age, endoscopic examination - Lund-Kennedy scoring system, CT Lund-Mackay scoring system, SNOT-22, asthma, and allergy status. AERD - aspirin-exacerbated respiratory disease. Statistical tests: Kruskal-Wallis (K-W) and $\mathrm{X}^{2}$ test. 
reaction product was obtained using 3,3'-diaminobenzidine tetrahydrochloride (Dako). Then, hematoxylin staining was performed in $1 \mathrm{~min}$. In the final stage, the sections were embedded in balsam and examined with light microscopy.

In order to obtain the most accurate results, the cells were counted by 2 independent specialists to avoid bias, and both scores were averaged and recorded. The color intensity was evaluated using the $\mathrm{H}$-score method. First, the results were recorded and assessed by a four-grade scale of staining intensity: 0 - none, 1 - weak, 2 - moderate, and 3 - strong for BRG1, BRM and BAF155 staining for each cell in a fixed field (100 cells). The H-score was based on predominant staining intensity. Using this method, the percentage of cells at each staining intensity level was calculated and, finally, a $\mathrm{H}$-score was assigned using the following formula:

$\mathrm{H}$-score $=[1 \times(\%$ cells 1$)+2 \times(\%$ cells 2$)+3 \times(\%$ cells 3$)]$

\section{Cell culture}

For the experiments, HNECs were grown in the following conditions: $37^{\circ} \mathrm{C}, 5 \% \mathrm{CO}^{2}$ and $90 \%$ humidity. When cells reached $80-90 \%$ confluence, media was removed and the cells were washed with phosphate-buffered saline (PBS; $37^{\circ} \mathrm{C}, \mathrm{pH} 7.4$ ) and fresh media containing LPS (SigmaAldrich Germany, Darmstadt, Germany; $5 \mu \mathrm{L} / \mathrm{mL}$ ), SEB (Staphylococcal Enterotoxin B; Sigma-Aldrich, St. Louis, USA; $1 \mu \mathrm{L} / \mathrm{mL}$ ), vitD3 (25-hydroxyvitamin D3 solution, Sigma-Aldrich Germany; $3 \mu \mathrm{L} / \mathrm{mL}$ ), or nothing (CG) were added to the cells and incubated for $24 \mathrm{~h}$. Moreover, the HNECs were stimulated by both vitD3 and LPS or SEB. After incubation, cells were collected and stored in $-80^{\circ} \mathrm{C}$ for further analysis.

\section{Quantitative reverse-transcription polymerase chain reaction}

Total RNA was isolated from the HNEC human nasal epithelial cells (PromoCell GmbH, Heidelberg, Germany) using RNA Isolation Kit (ReliaPrep RNA Cell Miniprep System; Promega, Madison, USA) according to the protocol. All procedures were conducted according to the manufacturers' instructions. The reverse transcriptase reaction was performed using Transcriptor First Strand cDNA Synthesis Kit (Roche, Basel, Switzerland). Expression of BRG1, BRM, BAF 155, BAF 170, and INI1 genes was measured with SybrGreen (BioRad, Hercules, USA) with UBIQUITIN as reference gene using following primers: (UBC-Fq ATTTGGGTCGCGGTTCTTG, UBC-Rq TGCCTTGACATTCTCGATGGT) for BRG1: reverse BRG1qR GCAACAGTACTGCCAGCAAC, forward BRG1qF GACATTCCAGTCTCGACCCC, for BRM: forward hBRMqFCGGTTTGATTGTGCCTGGTT, reversehBRMqR GCTTTTGTTCAGATCATAGAGCAT and for BAF 155: forward BAF155Fq GCCTGGCTTTCTCACTTCAC, reverse
BAF155Rq CTGAGGGTTTGAAAGGCAAA, for BAF170: forward BAF170Fq ACAGCAGAATGAACTCCGCT, reverse BAF170Rq GTCTGAGTGCTGCAGGTAGG, for INI1: forward INI1Fq GACCAGGACAGGAACACGAG, reverse INI1Rq CAAATGGAATGTGTGCCGG. Gene transcript levels of BRM, BRG-1, BAF 155, BAF 170, and INI1 were quantified using the ddCt method.

\section{Statistical analysis}

The data collected was saved in Microsoft Excel 2010 (Microsoft Corp., Redmond, USA) spreadsheet and analyzed using SAS v. 9.2 (SAS Intitute, Cary, USA). This allowed for a descriptive analysis, including averages, standard deviations (SD), medians, and lower and upper quartiles. In the first step, correlations between variables were calculated using Spearman's correlation coefficients. Several non-parametric tests were used in the analysis, such as Mann-Whitney U test, Kruskal-Wallis test (for many comparisons) and Wilcoxon test (comparison of 2 dependent data samples). We also used the $\chi^{2}$ test to compare discrete data. Several multiple regression models were used. Multiple regression presents in the tables the relations of the clinical and laboratory variables. In all statistical analyses, the level of significance was determined at the level of $\mathrm{p}<0.05 .^{22}$

\section{Results}

\section{The SWI/SNF protein expression in a tissue section}

Evaluation of the presence of SWI/SNF complex subunits showed that all proteins (BRG1, BRM, and BAF155) are expressed in the specimens. The stoichiometry in all groups was preserved. BRG1, BRM, and BAF155 were detected in all groups: CRSsNP group, CRSwNP group and CG (Fig. 1). Proteins were localized in the nuclei. The higher intensity of this staining was marked in CG and less intensive in patients with CRSsNP and CRSwNP.

\section{The SWI/SNF protein expression in CRSsNP group and CRSwNP group vs CG}

All the examined protein expression levels in the CG were significantly higher in comparison to patients with CRSwNP and CRSsNP (Fig. 2). We found no statistically significant difference in both CRS groups in regard to all subunits (BRG1, BRM and BAF155).

Moreover, we analyzed the SWI/SNF protein expression in patients treated with oral steroids during AMT and compared the results with those of patients not subject to this therapy in the CRSwNP group. We noticed differences for all SWI/SNF subunits in both parameters but without statistical significance (Fig. 3,4). 


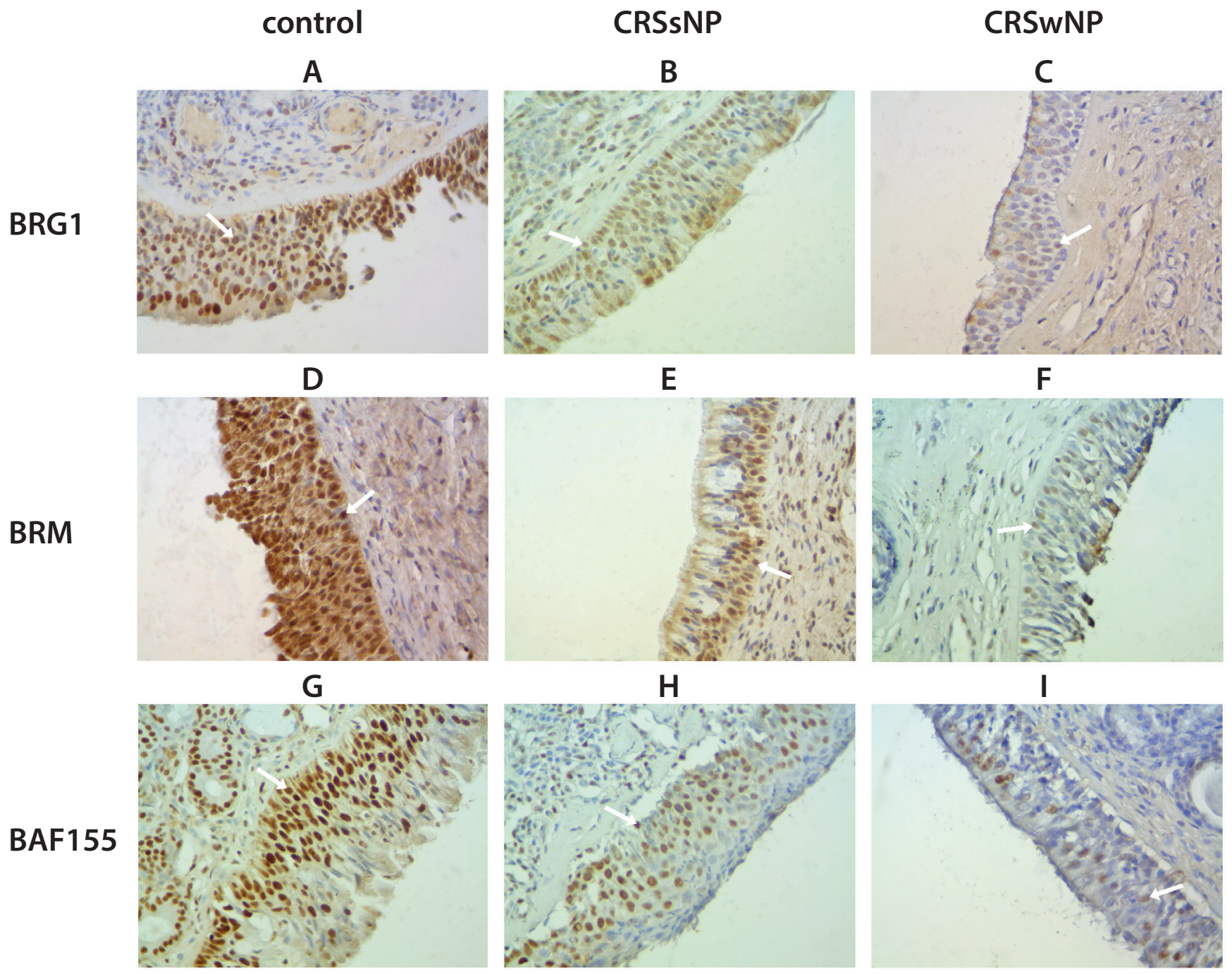

Fig. 1. Immunostaining of cells with BRG1 (A,B,C), BRM (D,E,F) and BAF155 (G,H,I) - antibodies in sinonasal epithelial cells. Dilution of the antibody: $1: 100$ - BRG1; 1:200 BRM; 1:200 BAF155

CRSsNP - chronic rhinosinusitis without nasal polyps; CRSwNP - chronic rhinosinusitis with nasal polyps. Magnification $\times 400$. The arrows show the nuclei after IHC staining.

\section{$\mathrm{H}$-score for the SWI/SNF correlations in patients with CRSwNP}

Furthermore, according to Spearman's test, we found a correlation between protein expression levels of BRG1, BRM and BAF155 in the CRSwNP group and other clinical parameters as follows. In the CRSwNP group, no correlations were observed.

Protein expression level of BRG1 in CRSwNP correlates inversely with serum level of eosinophils $(R=-0.3$; $\mathrm{p}<0.03$ ), neutrophils $(\mathrm{R}=-0.3 ; \mathrm{p}<0.03)$ (Fig. 5,6) and allergy $(\mathrm{R}=-0.3 ; \mathrm{p}<0.03)$.

Analyzes of association of BRM subunit with other clinical data in CRSwNP group displayed an inverse correlation of BRM protein level with allergy $(R=-0.3 ; \mathrm{p}<0.05)$.

The protein level of BAF155 correlates positively with VDR protein expression in CRSwNP group $(R=0.3$; $\mathrm{p}<0.043$ ) (Fig. 7).

\section{Results of multiple regression for the SWI/SNF subunits and other parameters}

The multiple regression analysis was performed in Stata v. 11.0 (StataCorp LLC, College Station, USA). The following clinical data was used in statistical models: allergy, asthma, white blood cell count, eosinophils blood count, neutrophils blood count, usage of oral steroids and nasal steroids, previous sinus surgery, SNOT-22 scale, L-M score, smoking, and H-score VDR.

In the CRSwNP group, allergy was the only factor significantly influencing BRM expression level $(\mathrm{F}=6.28$ and $\mathrm{p}=0.015)$. The patients with allergy had significantly lower BRM expression than patients in the CRSwNP group without allergy (Table 2).

Additionally, in the CRSwNP group, 2 other factors significantly influenced BRG1 expression: neutrophils and body 

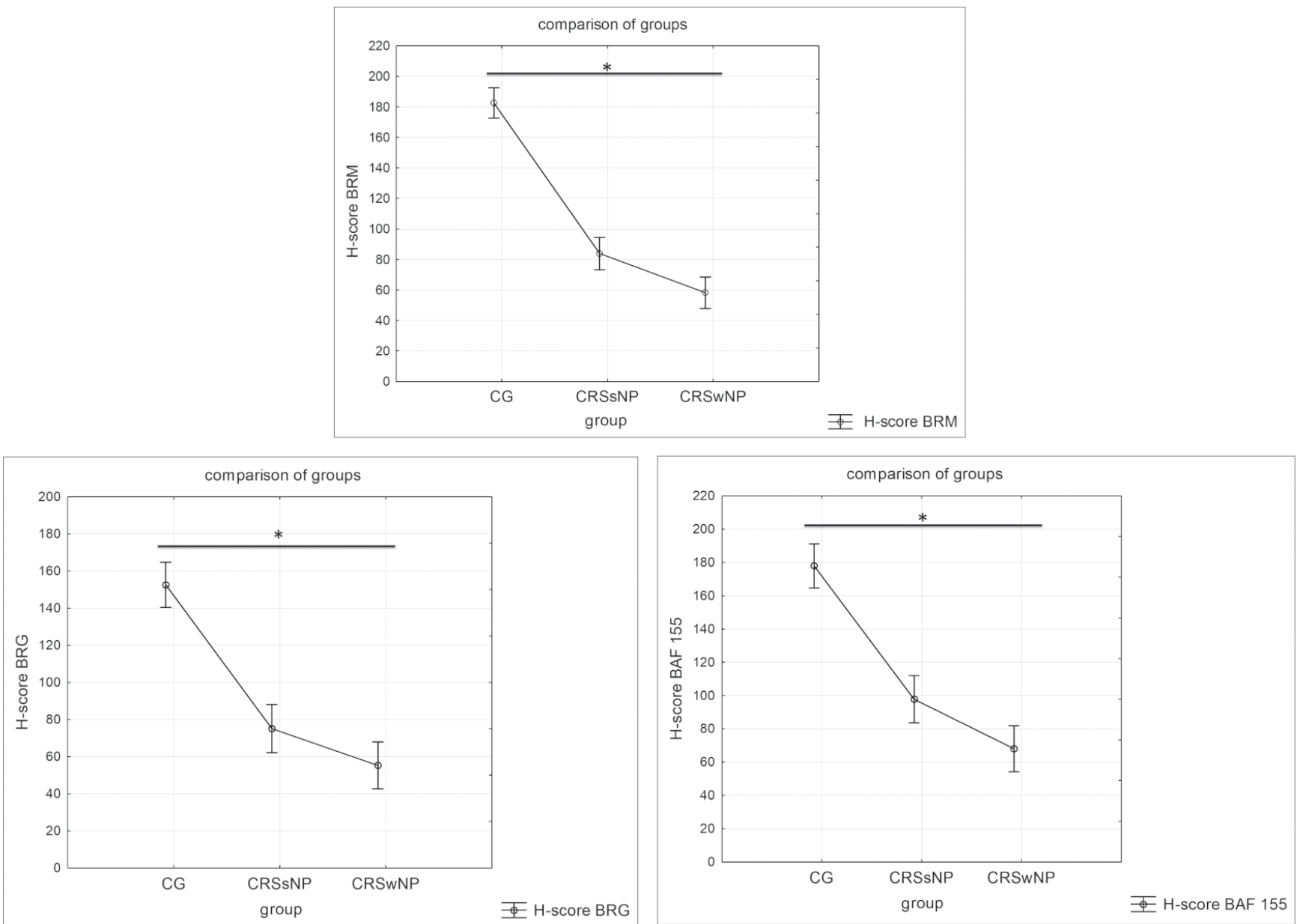

Fig. 2. Higher H-score for BRG1, BRM and BAF 155 staining intensity in control group (CG) in comparison to the CRSsNP group and the CRSwNP group (*p $<0.05$ ) CRSsNP - chronic rhinosinusitis without nasal polyps; CRSwNP - chronic rhinosinusitis with nasal polyps.

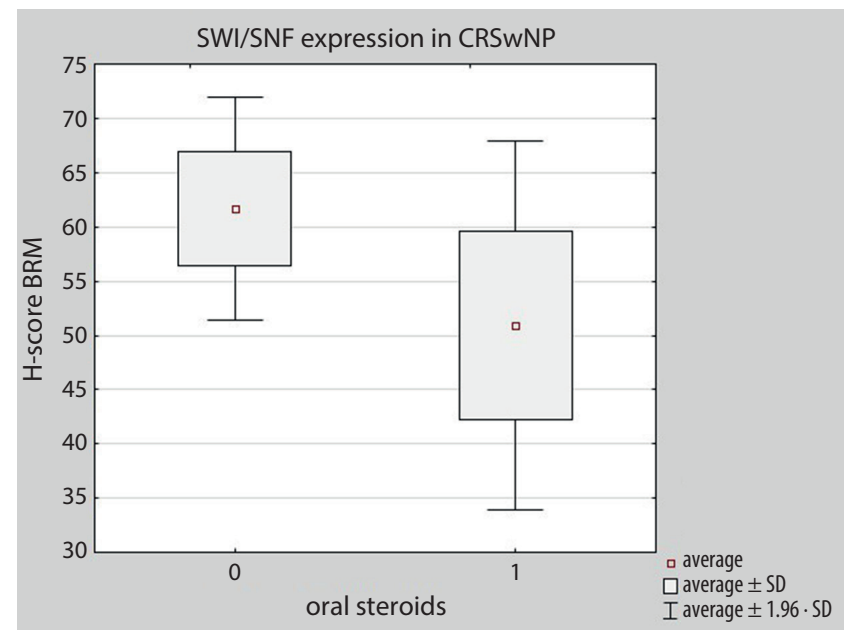

Fig. 3. Higher H-score for BRM staining intensity in CRSwNP - group of CRS with nasal polyps without oral steroids therapy (0) in comparison to patients with systemic treatment (1) $(p>0.05)$

CRSwNP - chronic rhinosinusitis with nasal polyps; CRS - chronic rhinosinusitis.

mass index (BMI). Patients with higher neutrophils blood count $(\mathrm{F}=9.23$ and $\mathrm{p}=0.004)$ had a significantly lower $\mathrm{H}$-score BRG1 (Table 2). Moreover, in the CRSwNP group, allergy

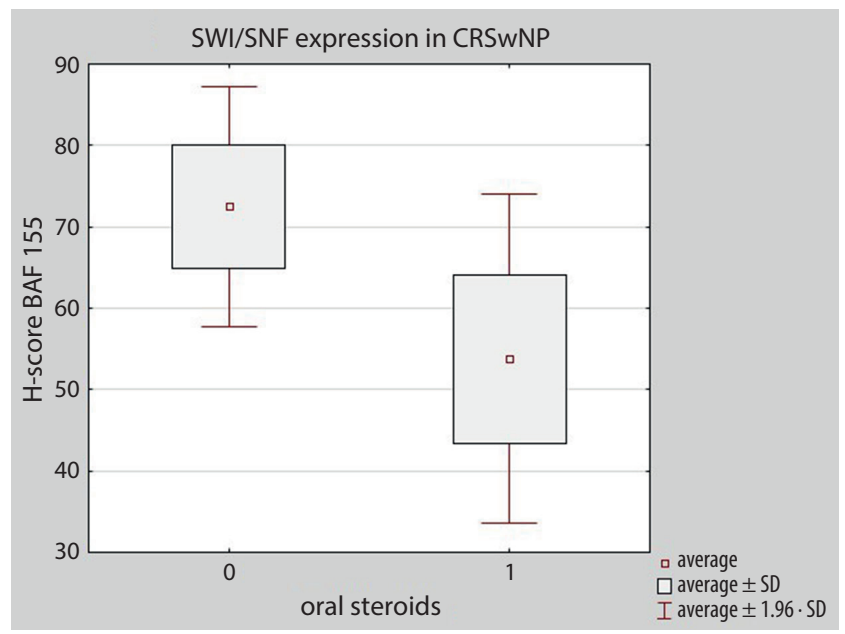

Fig. 4. Higher H-score for BAF 155 staining intensity in CRSwNP - group of CRS with nasal polyps without oral steroids therapy (0) in comparison to patients with systemic treatment (1) ( $p>0.05)$

CRSwNP - chronic rhinosinusitis with nasal polyps; CRS - chronic rhinosinusitis.

coexistence $(\mathrm{F}=4.12$ and $\mathrm{p}=0.048)$ and VDR expression level $(\mathrm{F}=4.19$ and $\mathrm{p}=0.048)$ significantly influenced BAF155 protein expression level $(\mathrm{F}=4.3$ and $\mathrm{p}=0.02)$ (Table 2$)$. 


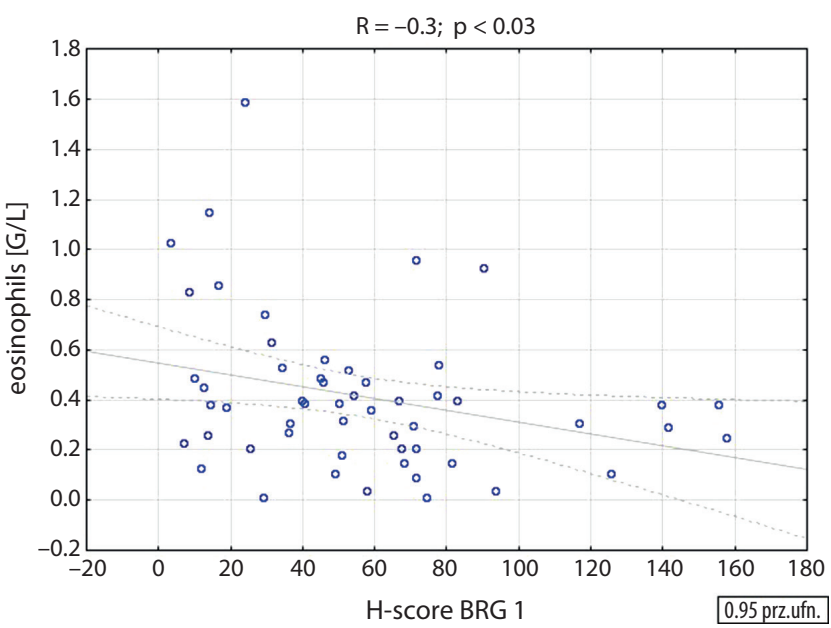

Fig. 5. Correlation of protein expression level of BRG1 with serum level of eosinophils ( $R=-0,3 ; p<0.03)$ in CRSwNP group

CRSwNP - chronic rhinosinusitis with nasal polyps.

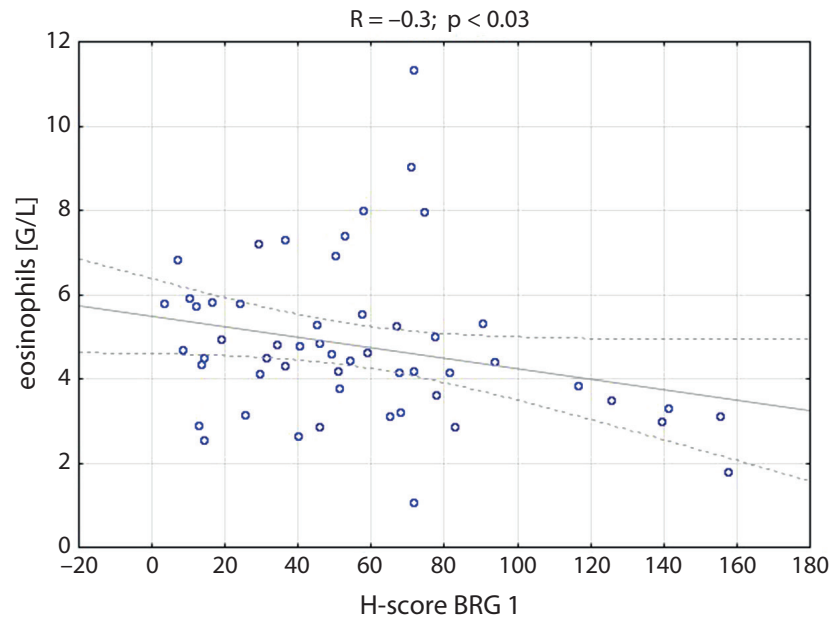

Fig. 6. Correlation of protein expression level of BRG1 with serum level of neutrophils ( $R=-0,3 ; p<0.03$ ) in CRSwNP group

CRSwNP - chronic rhinosinusitis with nasal polyps.

In the CRSsNP group, $\mathrm{L}-\mathrm{M}$ score was the only factor significantly influencing BRG 1 expression level $(\mathrm{F}=6.19$ and $\mathrm{p}=0.02)$. The patients with higher $\mathrm{L}-\mathrm{M}$ scores had

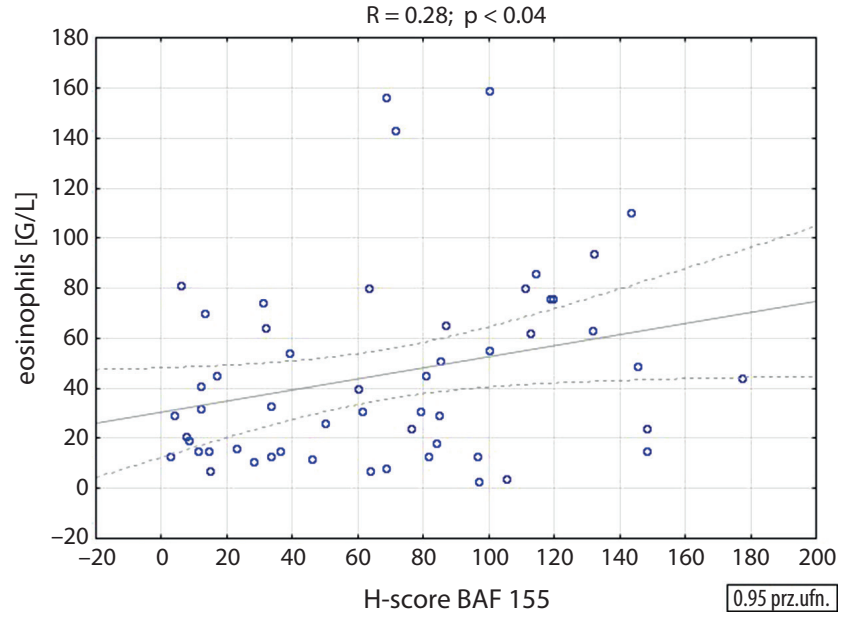

Fig. 7. The Spearman correlation for BAF155 protein expression level and VDR expression in CRSwNP group. BAF155 protein expression presented a positive correlation with VDR protein expression level $(p<0.05)$

CRSwNP - chronic rhinosinusitis with nasal polyps; VDR - vitamin D receptor.

a significantly lower BRG 1 expression than patients with lower L-M scores (Table 3).

\section{The SWI/SNF subunits expression in HNECs after treating with LPS, SEB and vitamin D3}

To examine if an expression of the SWI/SNF subunits encoding genes can be modulated by infection or vitD3, the HNEC cell line was stimulated with LPS, SEB and vitD3 for $24 \mathrm{~h}$. Moreover, HNECs were incubated with both vitD3 and LPS or SEB. The expression level of main SWI/SNF subunits was measured using quantitative reverse-transcription polymerase chain reaction (qRT-PCR) method with transcript-specific primers. Stimulation with LPS decreased significantly the transcript level for BRG1, BAF155 and INI1. The level of BAF 170 and INI1 increased after SEB stimulation. Moreover, the vitD3 and SEB stimulation of HNECs increased significantly the transcript level of BAF155 and BAF170, but decreased it for INI1. Increase of INI1 transcript level after vitD3 stimulation was higher for HNECs with LPS in comparison to HNECs without LPS. In the other cases, the transcript level for measured genes did not change (Fig. 8).

\section{Discussion}

In this study, we showed the SWI/SNF chromatin remodeling complex as an important factor that may influence steroids and vitamin D signaling pathways in sinonasal mucosa, thus contributing to the pathogenesis and treatment of CRS. The main findings of our study are the following: 1) higher 


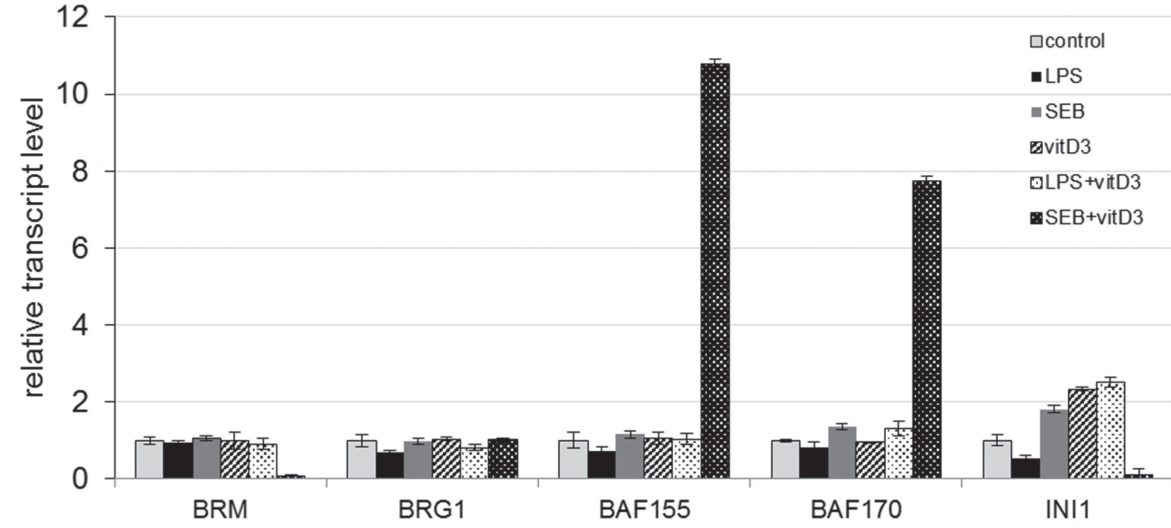

Table 3. The multiple regression analysis in chronic rhinosinusitis group without nasal polyps (CRSsNP) shows inverse correlation for BRG1 expression and Lund-Mackay CT score $(L-M)(p<0.05)$.

\begin{tabular}{|c|c|c|c|c|c|c|}
\hline \multirow{2}{*}{\multicolumn{2}{|c|}{ Variable }} & \multirow{2}{*}{$\begin{array}{l}\text { Parameter } \\
\text { estimate }\end{array}$} & \multirow{2}{*}{$\mathrm{F}$} & \multirow{2}{*}{$p$-value } & \multicolumn{2}{|c|}{ Model } \\
\hline & & & & & $F$ & p-value \\
\hline \multirow{2}{*}{ BRG 1} & intercept & 110.27 & 33.75 & $<0.001$ & \multirow{2}{*}{4.09} & \multirow{2}{*}{0.049} \\
\hline & $L-M$ & -5.14 & 4.09 & 0.049 & & \\
\hline
\end{tabular}

Fig. 8. Relative transcript level for the SWI/SNF main subunits BRG1, BRM, BAF155, BAF170 and INI1 after treating with LPS, SEB, vitD3, or both vitD3 and LPS or SEB compared with control untreated cells

LPS - lipopolysaccharide; SEB - staphylococcal enterotoxin B; vitD3 - vitamin D3. protein expression levels for BRG1, BRM and BAF155 in CG in comparison to CRSsNP group and CRSwNP group ( $<<0.05)$; 2) statistically significant negative correlation of the BRG1 protein expression with eosinophils and neutrophils in the CRSwNP group ( $<<0.05)$; 3) statistically significant positive correlation of the BAF155 protein expression with VDR expression level in the CRSwNP group $(\mathrm{p}<0.05)$; 4) relation in the multiple regression between the SWI/SNF protein expression level with allergy, neutrophils and BMI value $(\mathrm{p}<0.05)$ in CRS patients; 5) decreased transcript level of the SWI/SNF subunits in HNECs after LPS stimulation, but increased after SEB stimulation.

Many authors presented the important role of the SWI/SNF complex in the regulation of the inflammatory response and hormone metabolism. ${ }^{8,12,13}$ Ramirez-Carrozzi et al. showed that the SWI/SNF complex is involved in the inflammatory response stimulated with LPS murine macrophages. The constitutive association of BRG1 with the promoters of early primary inflammatory response genes suggests that the SWI/SNF complex might contribute to the initial establishment of "open" chromatin structures during macrophage development. ${ }^{6}$ Additionally, Hu et al. noticed that the SWI/SNF complex modulates the transactivation of the late-primary inflammatory response genes in macrophages in response to microbial challenges. The study showed the inhibition of the SWI/SNF complex recruitment to set gene promoter regions by lincRNA-Cox 2 siRNA-A in LPS-treated cells. ${ }^{23}$ According to the above data, we assumed that the SWI/SNF complex may play an important role in the inflammatory process contributing to CRS. Our results were based on the quantitative and qualitative analysis of the SWI/SNF complex core subunits expression in sinonasal mucosa. We observed a significantly higher protein expression level of the SWI/SNF subunits (BRG1, BRM and BAF155) in the CG compared to patients with CRS.

Therapy for CRS is currently based on both intranasal and oral GS. Oral steroids therapy is ordered in patients who manifest massive nasal polyps and worse prognosis in medical therapy. In this respect, it is important to analyze various aspects of GS activities and their interaction in relation to the SWI/SNF complex. In many cases, glucocorticoid therapy is ineffective; however, the reason remains unknown. It has been shown that proper functioning of GR is associated with the SWI/SNF complex. The SWI/SNF complex regulates genes expression following the response to GS. Hormones initiate a binding process of GR to glucocorticoid response element (GRE), in cooperation with the SWI/SNF complex, resulting in global changes in gene expression. Without the SWI/SNF complex, GR-dependent gene expression is blocked and GR function can be impaired. ${ }^{12,13,24}$ The decreased level of the main SWI/SNF subunits in CRS patients can suggest that steroid therapy can be inefficient (Fig. 9). The results of our study showed the lower expression of all examined SWI/SNF complex subunits in the CRSwNP group in comparison to CG $(\mathrm{p}<0.05)$. Moreover, we noticed higher SWI/SNF protein expression level in patients without oral steroids, though not statistically significant, in comparison to patients who underwent this treatment in the CRSwNP group ( $\mathrm{p}>0.05)$. Therefore, our results suggest that treatment with steroids in CRSwNP patients showing dysfunctional SWI/SNF signaling may not be effective. In consequence, the alternative medical treatment should be proposed.

To better identify recalcitrant patients who are unresponsive to steroids therapy, we analyzed the SWI/SNF subunits and other clinical data. We found that the BRG1 and ATPase SWI/SNF subunit abundance presented 
A

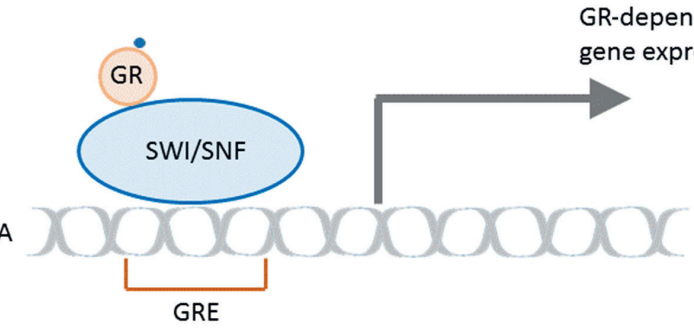

B

DNA

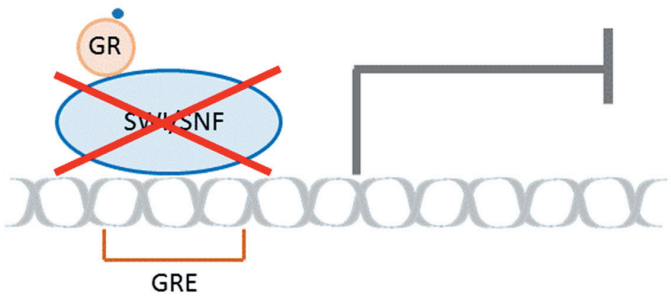

C

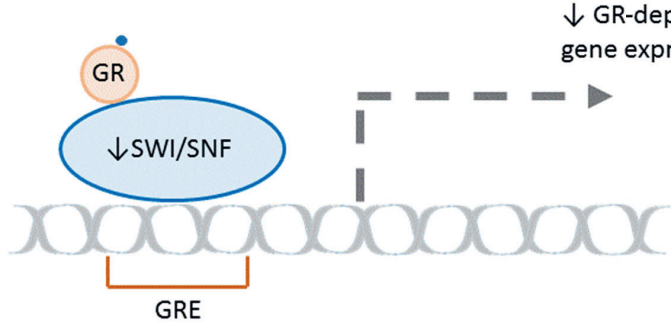

Fig. 9. A model describing GR and the SWI/SNF complex cooperation in response to steroids treatment. Hormone (small blue dot) is shown binding the GR. It enters the nucleus and binds target sequences, such as the GRE to the DNA. Next, the complex SWI/SNF cooperates with the promoter through interaction with $G R$, and then the nucleosomes are repositioned. A. The SWI/SNF complex is correct, proper GR response gene expression. B. Loss of the SWI/SNF complex, blocked GR-dependent gene expression. C. The SWI/SNF complex deficiency, reduced GR-dependent gene expression

GR - glucocorticoid receptor; GRE - glucocorticoid response element.

an inverse correlation with the number of eosinophils and neutrophils in the blood of patients with CRSwNP. For a better understanding of the disease course and prediction of treatment outcomes, some authors categorized CRSwNP into various subtypes, such as eosinophilic CRSwNP, neutrophilic CRSwNP and noneosinophilic nonneutrophilic CRSwNP. ${ }^{25,26}$ Other data indicated that blood eosinophilia is related to the extent of sinonasal mucosal involvement, the severity of nasal disease, size of nasal polyps, and a higher risk of disease recurrence. ${ }^{27,28}$ Moreover, Fokkens et al. proposed another form of management for patients with eosinophilic CRS. ${ }^{29}$ Our study showed lower expression of BRG1 in the CRSwNP group with a higher level of blood eosinophils and neutrophils in comparison to CRSwNP individuals without eosinophilia and neutrophilia. Therefore, this negative correlation of eosinophils and neutrophils with the SWI/SNF complex may expound the worse prognosis in this group of patients. Thus, blood analysis of the amount/number of eosinophils and neutrophils could be a prognostic factor for GR effectiveness through the SWI/SNF complex abundance. In the group of patients with CRSwNP and eosinophilia/neutrophilia nasal steroids, there will probably be poor effectiveness and some treatment modification will be needed. These findings, i.e., high number of eosinophils and neutrophils, might be an efficient and simple clinical marker to identify patients with CRSwNP who present impaired SWI/SNF expression, meaning that they either respond worse or not at all to medical treatment with oral steroids. To our knowledge, this is the first report on the SWI/SNF complex subunits expression in CRS in relation to the treatment.

Further, we analyzed the correlations of our results with VDR expression. Our previous study on CRS pathophysiology presented VDR and $1 \alpha$-hydroxylase expression in sinonasal mucosa. This study showed a statistically significant decrease of VDR expression in CRSsNP and CRSwNP patients in comparison to the CG. ${ }^{30}$ Additionally, Wei et al. analyzed the role of the SWI/SNF complex (BAF and PBAF) subunits in relation to VDR and vitamin $D$ in pancreatic $\beta$-cells protection. Ligand binding promotes VDR association with the SWI/SNF subunit, resulting in an anti-inflammatory response in the murine type 2 diabetes model. ${ }^{14}$ Likewise, VDR signaling plays an important role in the regulation of the immune processes in paranasal sinuses. ${ }^{15-19}$ In our study, the protein expression level of BAF155, a core SWI/SNF chromatin remodeling complex subunit, correlates positively with VDR expression level in the CRSwNP group. Further, after administration of vitD3 to the HSNEC, we observed a significant increase in the BAF170 and INI1 transcript level. Therefore, we assume that VDR, upon ligand- (vitD3) binding, may reduce the pro-inflammatory response in sinonasal mucosa via the SWI/SNF-induced transcriptional changes, thus contributing to the treatment of CRS.

Furthermore, in multiple regression models, we found the relation of the SWI/SNF subunits with numerous clinical findings, i.e., L-M scores, allergy, BMI values, neutrophils, and VDR abundance. The BRG1 protein expression level was associated with $\mathrm{L}-\mathrm{M}$ score. The $\mathrm{L}-\mathrm{M}$ CT score is a useful tool to assess the radiological stage of rhinosinusitis. ${ }^{31}$ Our results confirm lower BRG1 expression in CRSsNP patients with higher L-M scores. In the CRSwNP group, BRM expression level was associated with allergy. The expression of BRG1 protein is associated with the amount of neutrophils in blood and BMI value in the CRSwNP group. Additionally, interesting results were observed for BAF155 protein level, e.g., its relation with allergy and VDR abundance. Therefore, we showed that there might be a link between allergy, vitamin $\mathrm{D}$ and VDR in the CRS pathophysiology. ${ }^{15-19}$ Our results suggest a new mechanism of VDR action in cooperation with the SWI/SNF chromatin remodeling complex.

Moreover, we analyzed the SWI/SNF genes expression after HNECs line has been treated with vitD3, LPS, SEB, and both vitD3 with LPS or SEB. The vitD3 treatment increased the INI1 encoding gene transcript level. The SEB had an increased BAF170 and INI1 genes expression. 
The LPS treatment of HNECs decreased the expression of BRG1, BAF155 and INI1 genes; however, it did not affect $B R M$ and BAF170 genes transcription. The vitamin D and SEB stimulation of HNECs significantly increased BAF155 and BAF170 transcript level. The role of vitamin D and its receptors has been vastly addressed in recent studies on CRS pathophysiology. Vitamin D is recognized as the factor influencing the CRS. ${ }^{3}$ Research results from the last decade show the immunomodulatory effect of vitamin D on the mechanisms of CRS by reducing inflammation, ${ }^{32,33}$ inducing cathelicidin (hCAP18), which is the only antimicrobial peptide produced by the human body, ${ }^{34}$ and by stimulating neutrophils and macrophages in anti-inflammatory responses. ${ }^{35}$ Other studies on SEB have demonstrated that SEB lead to T-cell proliferation (CD4+ and $\mathrm{CD} 8+$ ) and pro-inflammatory cytokine production, and can influence the activity of immunomodulatory and pro-inflammatory effector epithelial cells and, therefore, may have a potentially important role in the pathogenesis of CRS. The presence of nasal polyps in CRS seems to be associated with inflammatory mechanisms resulting from microbial products. ${ }^{36-38}$ The LPS is a cell wall surface antigen of Gram-negative bacteria and a biologically active substance activating many transcription factors. It triggers an inflammatory signaling cascade. ${ }^{39}$ Therefore, SEB and LPS may contribute to CRS pathogenesis through respectively increasing or decreasing gene expression encoding for the SWI/SNF complex subunits, subsequently compromising anti-inflammatory signaling pathways of steroids, thus contributing to recalcitrant course of the disease.

The SWI/SNF interacts with GR and regulates genes expression following the response to GS. Without the SWI/SNF complex, GR-dependent gene expression is blocked and GR function can be impaired. ${ }^{12,13,24}$ Additionally, as a continuation of our previous studies, ${ }^{30}$ we found a link (positive correlation) between VDR and the SWI/SNF complex in patients with CRSwNP. Moreover, GR and vitD3 presents antagonistic activity in human cells signaling. ${ }^{40}$ Due to the fact that the SWI/SNF complex may be involved in both GR and VDR signaling pathways, it is very likely that decreased expression of this complex in the sinonasal mucosa of CRSwNP patients can disturb anti-inflammatory function of steroids because of competition with vitD3 in the sinonasal mucosa contributing to CRS. Results of our study are of clinical relevance due to the fact that based on the correlation of the SWI/SNF proteins expression and clinical data, it is possible to identify responders and non-responders to steroids treatment in the CRSwNP group.

\section{Conclusions}

The strength of this study is that it included an analysis of a large group of patients with CRS with or without polyps compared to CG. Furthermore, this is the first report on the expression of the SWI/SNF complex subunits in the sinonasal mucosa and its clinical associations. We proved the significant differences in the protein expression of the SWI/SNF subunits in the sinonasal mucosa between the groups. We found a positive correlation for BAF155 protein expression with VDR level and a negative correlation of BRG1 subunit with blood eosinophils and neutrophils. Moreover, we analyzed the expression of genes encoding for SWI/SNF subunits in HNECs after vitD3, LPS and SEB stimulation. Our results contributed to the knowledge of the molecular inflammatory process in sinonasal mucosa in the CRS and showed the need for alternative treatment options in recalcitrant CRS. Further analysis of the function of the SWI/SNF complex in response to steroids and vitamin D may be beneficial for an understanding of the pathophysiology of CRS, especially in patients with a recalcitrant course of the disease.

\section{ORCID iDs}

Katarzyna Kowalik (D) https://orcid.org/0000-0002-9238-2266 Martyna Waniewska-Łęczycka

(D) https://orcid.org/0000-0002-9002-2099

Elżbieta Sarnowska (D) https://orcid.org/0000-0001-7723-1198

Natalia Rusetska (D) https://orcid.org/0000-0001-7496-2489

Janusz Sierdziński (D) https://orcid.org/0000-0001-7395-9050

Mariola Zagor (D) https://orcid.org/0000-0002-9181-950X

\section{References}

1. Bachert C, Holtappels G. Pathophysiology of chronic rhinosinusitis: Pharmaceutical therapy options. GMS Curr Top Otorhinolaryngol Head Neck Surg. 2015;14:Doc09.

2. Fokkens WJ, Lund VJ, Mullol J, et al. EPOS 2012: European position paper on rhinosinusitis and nasal polyps 2012. A summary for otorhinolaryngologists. Rhinology. 2012;50(1):1-12.

3. Orlandi RR, Kingdom TT, Hwang PH, et al. International Consensus Statement on Allergy and Rhinology: Rhinosinusitis. Int Forum Allergy Rhinol. 2016;6(Suppl 1):S22-209.

4. Akdis CA, Bachert C, Cingi $C$, et al. Endotypes and phenotypes of chronic rhinosinusitis: A PRACTALL document of the European Academy of Allergy and Clinical Immunology and the American Academy of Allergy, Asthma \& Immunology. J Allergy Clin Immunol. 2013;131(6): 1479-1490.

5. Ball SL, Suwara MI, Borthwick LA, Wilson JA, Mann DA, Fisher AJ. How reliable are sino-nasal cell lines for studying the pathophysiology of chronic rhinosinusitis? Ann Otol Rhinol Laryngol. 2015;124(6): 437-442.

6. Ramirez-Carrozzi VR, Nazarian AA, Li CC, et al. Selective and antagonistic functions of SWI/SNF and Mi-2beta nucleosome remodeling complexes during an inflammatory response. Genes Dev. 2006;20(3): 282-296.

7. Santen GW, Kriek M, van Attikum H. SWI/SNF complex in disorder: Switching from malignancies to intellectual disability. Epigenetics. 2012;7(11):1219-1224.

8. Sarnowska E, Gratkowska DM, Sacharowski SP, et al. The role of SWI/SNF chromatin remodeling complexes in hormone crosstalk. Trends Plant Sci. 2016;21(7):594-608.

9. Smith CL, Horowitz-Scherer R, Flanagan JF, Woodcock CL, Peterson CL. Structural analysis of the yeast SWI/SNF chromatin remodeling complex. Nat Struct Biol. 2003;10(2):141-145.

10. Cairns BR, Kim YJ, Sayre MH, Laurent BC, Kornberg RD. A multisubunit complex containing the SWI1/ADR6, SWI2/SNF2, SWI3, SNF5, and SNF6 gene products isolated from yeast. Proc Natl Acad SciUSA. 1994;91(5):1950-1954.

11. Lou H, Wang C, Zhang L. Steroid transnasal nebulization in the treatment of chronic rhinosinusitis. Curr Opin Allergy Clin Immunol. 2016; 16(1):39-44. 
12. Trotter KW, King HA, Archer TK. Glucocorticoid receptor transcriptional activation via the BRG1-dependent recruitment of TOP2beta and Ku70/86. Mol Cell Biol. 2015;35(16):2799-2817.

13. King HA, Trotter KW, Archer TK. Chromatin remodeling during glucocorticoid receptor regulated transactivation. Biochim Biophys Acta. 2012;1819(7):716-726.

14. Wei Z, Yoshihara $E$, He N, et al. Vitamin D switches BAF complexes to protect beta cells. Cell. 2018;173(5):1135-1149.e1115.

15. Stokes PJ, Rimmer J. The relationship between serum vitamin D and chronic rhinosinusitis: A systematic review. Am J Rhinol Allergy. 2016; 30(1):23-28.

16. Frieri M, Kumar K, Boutin A. Review: Immunology of sinusitis, trauma, asthma, and sepsis. Allergy Rhinol (Providence). 2015;6(3):205-214.

17. Shahangian A, Schlosser RJ. Role of vitamin D in pathogenesis of chronic sinusitis with nasal polyposis. Adv Otorhinolaryngol. 2016;79: 86-90.

18. Mostafa Bel D, Taha MS, Abdel Hamid T, Omran A, Lotfi N. Evaluation of vitamin D levels in allergic fungal sinusitis, chronic rhinosinusitis, and chronic rhinosinusitis with polyposis. Int Forum Allergy Rhinol. 2016;6(2):185-190.

19. Khalid AN, Ladha KS, Luong AU, Quraishi SA. Association of vitamin D status and acute rhinosinusitis: Results from the United States National Health and Nutrition Examination Survey 2001-2006. Medicine (Baltimore). 2015;94(40):e1447.

20. Horak F, Doberer D, Eber E, et al. Diagnosis and management of asthma: Statement on the 2015 GINA Guidelines. Wien Klin Wochenschr. 2016;128(15-16):541-554.

21. Psaltis AJ, Li G, Vaezeafshar R, Cho KS, Hwang PH. Modification of the Lund-Kennedy endoscopic scoring system improves its reliability and correlation with patient-reported outcome measures. Laryngoscope. 2014;124(10):2216-2223.

22. Lang TA, Secic M. How to Report Statistics in Medicine. Philadelphia, PA: American College of Physicians; 2006:490.

23. Hu G, Gong AY, Wang Y, et al. LincRNA-Cox2 promotes late inflammatory gene transcription in macrophages through modulating SWI/SNF-mediated chromatin remodeling. J Immunol. 2016;196(6): 2799-2808.

24. Muratcioglu S, Presman DM, Pooley JR, et al. Structural modeling of GR interactions with the SWI/SNF chromatin remodeling complex and C/EBP. Biophys J. 2015;109(6):1227-1239.

25. Ikeda K, Shiozawa A, Ono N, et al. Subclassification of chronic rhinosinusitis with nasal polyp based on eosinophil and neutrophil. Laryngoscope. 2013;123(11):E1-9.
26. Tecimer SH, Kasapoglu F, Demir UL, Ozmen OA, Coskun H, Basut O. Correlation between clinical findings and eosinophil/neutrophil ratio in patients with nasal polyps. Eur Arch Otorhinolaryngol. 2015;272(4): 915-921.

27. Hu Y, Cao PP, Liang GT, Cui YH, Liu Z. Diagnostic significance of blood eosinophil count in eosinophilic chronic rhinosinusitis with nasal polyps in Chinese adults. Laryngoscope. 2012;122(3):498-503.

28. Sreeparvathi A, Kalyanikuttyamma LK, Kumar M, Sreekumar N, Veerasigamani N. Significance of blood eosinophil count in patients with chronic rhinosinusitis with nasal polyposis. J Clin Diagn Res. 2017;11(2):MC08-MC11.

29. Fokkens WJ, Reitsma S. Proposal for an algorithm on the management of chronic rhinosinusitis. Allergy. 2019;74(7):1415-1416.

30. Tomaszewska M, Sarnowska E, Rusetska N, et al. Role of vitamin D and its receptors in the pathophysiology of chronic rhinosinusitis. J Am Coll Nutr. 2019;38(2):108-118.

31. Lund VJ, Mackay IS. Staging in rhinosinusitis. Rhinology. 1993;31(4): 183-184.

32. Hariri BM, Cohen NA. New insights into upper airway innate immunity. Am J Rhinol Allergy. 2016;30(5):319-323.

33. Akbar NA, Zacharek MA. Vitamin D: -mmunomodulation of asthma, allergic rhinitis, and chronic rhinosinusitis. Curr Opin Otolaryngol Head Neck Surg. 2011;19(3):224-228.

34. Dimeloe S, Nanzer A, Ryanna K, Hawrylowicz C. Regulatory T cells, inflammation and the allergic response: The role of glucocorticoids and vitamin D. J Steroid Biochem Mol Biol. 2010;120(2-3):86-95.

35. Kamen DL, Tangpricha V. Vitamin D and molecular actions on the immune system: Modulation of innate and autoimmunity. J Mol Med (Berl). 2010;88(5):441-450.

36. Tripathi A, Kern R, Conley DB, et al. Staphylococcal exotoxins and nasal polyposis: Analysis of systemic and local responses. Am J Rhinol. 2005;19(4):327-333.

37. Schleimer RP. Immunopathogenesis of chronic rhinosinusitis and nasal polyposis. Annu Rev Pathol. 2017;12:331-357.

38. Bachert C, Gevaert P, van Cauwenberge P. Staphylococcus aureus enterotoxins: A key in airway disease? Allergy. 2002;57(6):480-487.

39. van der Merwe R, Molfino NA. Challenge models to assess new therapies in chronic obstructive pulmonary disease. Int J Chron Obstruct Pulmon Dis. 2012;7:597-605.

40. Obradovic D, Gronemeyer H, Lutz B, Rein T. Cross-talk of vitamin D and glucocorticoids in hippocampal cells. J Neurochem. 2006;96(2): 500-509. 